\title{
Nitric oxide: a key driver of signaling in plants
}

\begin{abstract}
The colorless gaseous compound nitric oxide (NO) is a lipophilic free radical that diffuses readily through the plasma membrane. These attributes make nitric oxide ideal for a transient paracrine (between adjacent cells) and autocrine (within a single cell) signaling molecule. The half-life of NO in biological tissues is estimated to be $<$ $6 \mathrm{Sec}$. This short half-life reflects the highly reactive nature of NO. It reacts directly with metal complexes and other radicals and indirectly as a reactive nitrogen oxide species with DNA, protein, and lipids. NO was first described in 1772 as nitrous air by Joseph Priestly the English theologian chemist and by co-educators. He was also the first to describe nitrous oxide (N2O), which he named nitrous air diminished. Priestly' nitrous air induced a sensation of mild drunkenness, often coupled with bouts of uncontrollable laughter.
\end{abstract}

Volume 3 Issue 3 - 2018

\author{
Prasann Kumar,' Shweta Pathak ${ }^{2}$ \\ 'Department of Agronomy, Assistant Professor, Lovely \\ Professional University, India \\ ${ }^{2}$ School of Biochemistry, Devi Ahilya Vishwavidyalaya, India
}

Correspondence: Prasann Kumar, Assistant Professor, Department of Agronomy, School of Agriculture, Lovely Professional University, Jalandhar, I 444 I I, Punjab, India, Email prasann0659@gmail.com; prasann.21784@Ipu.co.in

Received: November II, 2017 | Published: May 18, 2018

Keywords: agriculture, biotic, cadmium, diffuse, nitric oxide

\section{Introduction}

Fast forward to the most recent time, NO has been demonstrated to orchestrate a plethora of physiological function in mammals, was the subject of the Nobel Prize in 1998 and was named molecule of the year in 1992 by journal of science. Despite of animal it was first reported in plant in 1779 by Klepper. ${ }^{1}$ Almost two decades later by the late 1990s, plant biologists had started to pay attention to NO playing role in plant immunity, initially in potato (Solanum tuberosum) ${ }^{2-4}$ and then two years later in Arabidopsis..$^{5-7}$ However the Era is now gathering substantial momentum. It was undoubtedly an era of great enthusiasm for $\mathrm{NO}$ as a plant signaling molecule and today it is known to play a crucial role in the regulation of physiological process ranging from development to adaptation to biotic ${ }^{8-10}$ and abiotic stress. ${ }^{11}$ Furthering the application of new tools and technologies to study and addressing the molecular mechanisms employed by NO to control a variety of key cellular processes. ${ }^{12,13}$ Nitric oxide has a potent skill to encroach on a noticeable plant world due its enormous owndom like free radical, small size, no charge, short lived and permeability across biological membranes. It has also regulated signaling pathway for oxidative stress, mitochondrial activity, programmed cell death, ${ }^{14,15}$ growth and development in plants and responsible for the guidance of markable spectrum of plant cellular mechanism. The role of NO in plant may be equally diverse. The participation of $\mathrm{NO}$ in plant disease resistance pathways has been reported on several occasions ${ }^{5,6,16,17}$ and role of $\mathrm{NO}$ in the abscisic acid (ABA) signal transduction pathway leading to stomatal closure has been also demonstrated..$^{18}$ The last decades, the role of No in tolerance of abiotic stress has established much consideration, it has begun to emerge as an important endogenous signaling molecule in the adaptation of plant to abiotic stress. As it is evident from the present review, recent progress on NO potentiality intolerance of plants to environmental stresses has been impressive. ${ }^{19}$ These investigations suggest that, NO it possesses antioxidant activity and might act as a signaling in activating ROI-scavenging enzyme activities under abiotic stress. NO responses to stress due to salt, drought, temperature, UV-B and heavy metal, which in their extreme limit responsible for cause of serious threats and set the plants with impaired growth, physiological and biological activities that are witnessed by lost in crop growth and yield etc. This review represents attention to the description of numerous NO sources, synthesis, the myriad role of $\mathrm{NO}$ and the molecular mechanism underpinning their function. Nonenzymatic NO production, according to the equation should occur only at $\mathrm{pH}$ below 4.5 , since the $\mathrm{pKa}$ of nitrous acid is about 3.2.

$$
2 \mathrm{HNO}_{2} \leftrightarrow \mathrm{NO}+\mathrm{NO}_{2}+\mathrm{H}_{2} \mathrm{O} \leftrightarrow 2 \mathrm{NO}+2 \mathrm{O}_{2}+\mathrm{H}_{2} \mathrm{O}
$$

NO is involved in plant metabolism and the nitrification and denitrification cycle provides $\mathrm{NO}$ as a byproduct of nitrous oxide oxidation into the environment by mean of a non-enzymatic mechanism..$^{20}$ The studies of NO on plant metabolism date back to the 1960s when Fewson et al. ${ }^{21}$ address the recruitment of NO by microorganisms and higher plants. NO was suggested to be the key intermediate in the metabolism of inorganic nitrogen compound in higher plants and nitrogen fixing organisms. It was only in 1994 that NO was proved to be endogenously produced in a non-enzymatic way through conversion of nitrogen dioxide to NO by carotenoids in the light. ${ }^{22}$ Several plant systems use nitrite as a substrate, according to the basic reaction.

$$
\mathrm{NO}_{2}^{-}+\mathrm{e}^{-}+2 \mathrm{H}^{+} \rightarrow \mathrm{NO}+\mathrm{H}_{2} \mathrm{O}
$$

$$
2 \mathrm{HNO}_{2} \leftrightarrow \mathrm{NO}+\mathrm{NO}_{2}+\mathrm{H}_{2} \mathrm{O} \leftrightarrow 2 \mathrm{NO}+2 \mathrm{O}_{2}+\mathrm{H}_{2} \mathrm{O}
$$

Moreover synthesis of NO on the apoplast has also been described by a non-enzymatic mechanism, whereby nitrite is converted to NO under acidic conditions in response to abscisic acid and gibberellins. ${ }^{23}$ These are also like Cytosolic NR (cNR), A plasma membranebound NR (PM-NR) associated with a PM- nitrite: NO reductase, Mitochondrial electron transport, Xanthine dehydrogenase/oxidase, Non-enzymatic NO formation at acidic pH. Nitric oxide is produced by a group of enzymes called nitric oxide syntheses. These enzymes convert arginine into citrulline, producing $\mathrm{NO}$ in the process. Oxygen and NADPH are necessary co-factors. ${ }^{24}$ There are three isoforms of nitric oxide synthase (NOS) named according to their activity or the tissue type in which they were first described. The isoforms of NOS are neuronal NOS (or nNOS), endothelial NOS (or eNOS) and inducible 
NOS (or iNOS) ${ }^{25}$ These enzymes are also sometimes referred to by number, so that nNOS is known as NOS1, iNOS is known as NOS2 and eNOS is NOS.3.Despite the names of these enzymes, all three isoforms can be found in a variety of tissues and cell types (Figure 1).

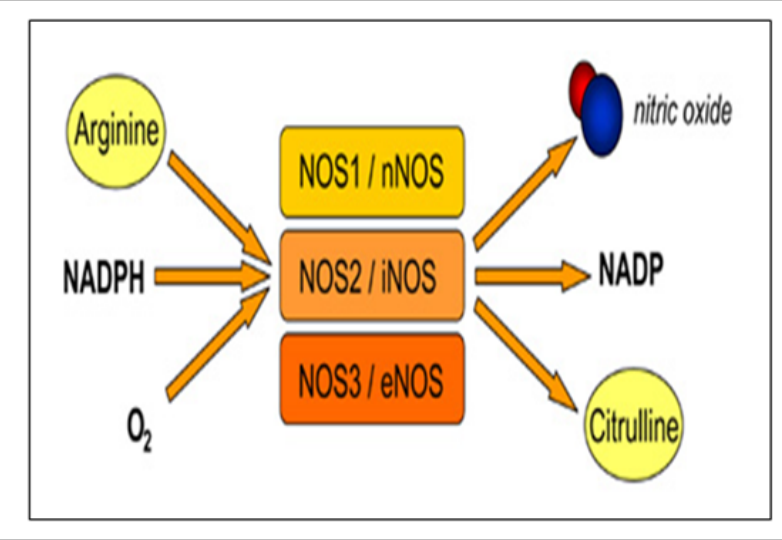

Figure I Oxidative pathway of nitrous oxide syntheses.

Source: http://www.reading.ac.uk/nitricoxide/intro/no/synthesis.htm.

Both enzymatic and non-enzymatic pathways have been described, but there is no consensus in sight on central source of NO in plant, and even less its regulation. NOS isoform may also present in the mitochondria, including a constitutive (cmtNOS) and inducible (imtNOS) mitrochandrial $\operatorname{NOS}^{26,27}$ which are thought to derive from cytosolic nNOS and INOS, respectively. Some studies have failed to locate mtNOS isoform, which could be related to different experimental designs or methods utilized in the NOS activity assays. NOS proteins catalyze the NADP-dependent oxidation of arginine (Arg) to NO and Citrulline. However, genes encoding a structurally related enzyme have not been identified in higher plants despite the completion of numerous genome projects. Oxidative mechanisms include the production of NO from $\mathrm{L}$-arginine ( $\mathrm{L}-\mathrm{Arg}$ ), polyamines or hydroxylamines. By contrast, reductive routes are dependent upon nitrite as the primary substrate and include reduction via NADPH nitrate reductase (NR), a cytosolic enzyme associated with nitrogen assimilation, whose primary function is the reduction of nitrate to nitrite ${ }^{28}$ it can further reduce nitrite to $\mathrm{NO}$ by the mitochondrial electron transport-dependent reductase, ${ }^{29}$ Which uses arginine as a substrate.

\section{Oxidative pathway of no synthesis}

There are three different isoforms of the NOS which classify on their localization Zhou et al. ${ }^{30}$ All NOS are active as homodimers converting $\mathrm{L}$-arginine to $\mathrm{L}-$ citrulline and NO.

$$
\mathrm{L}-\operatorname{arginine}+\mathrm{NAD}(\mathrm{P}) \mathrm{H}+\mathrm{O}_{2} \rightarrow \mathrm{L}-\text { citrulline }+\mathrm{NAD}(\mathrm{P})++\mathrm{H}_{2} \mathrm{O}+\mathrm{NO}
$$

When the availability of L-arginine is reduced, these enzymes also produce superoxide anion and NO, which may create peroxynitrite. Also a loss of function mutant, no overproducer 1(nox 1) has been reported to have several fold greater concentration of $\mathrm{L}$-arginine and this plant line exhibits excessive NO and citrulline accumulation.
These data therefore imply the existence of a plant NOS like enzyme. Further, numerous studies in both Arabidopsis and tobacco (Nicotiana tobacum) have implicated, such NOS like activities as the source of reactive nitrogen intermediates during the nitrosative burst associated with the plant immune function. ${ }^{5,6} \mathrm{~A}$ recent paper reported the presence of NOS in a single celled green algae Osteroccocus tauri. This algae NOS possessed $45 \%$ similarity to human NOS. This enzyme exhibited NOS activity in vitro and possessed similar property to animal NOS proteins in the terms of the $\mathrm{km}$ for $\mathrm{L}$-arginine (12microM) and the rate of NADPH oxidation. Unfortunately, these genes to date, no direct ortholog seem to be present in Arabidopsis and higher plants. ${ }^{31}$ In the late 1990s, the palate of available tools to dissect NOS production was composed by several macrophagic NOS compound inhibitors such as $\mathrm{N}^{\mathrm{G}}$ monomethyl L-arginine (NMMA) and arginine analogs, and assay for arginine to citrulline conversion were used to detect the presence of NOS like enzymes in the different plant tissues (e.g. root, leaves, stems) and organelles (e.g. peroxisome). ${ }^{6,17,32}$ Interestingly, increase the concentration of polyamines, spermine and spermidine induce NO release, but the actual reaction mechanism still not resolved. Polyamines mediated NO production has been thought to be involved in root development and embryogenesis ${ }^{33}$ cadmium toxicity ${ }^{34}$ and drought stress. ${ }^{35}$ Hydroxylamine mediated NO synthesis is also a potent source of NO synthesis while location still unclear. Hydroxylamine and Reactive oxygen intermediate is the substrate for NO synthesis. According to given hypothesis this pathway involved in regulation of ROI concentration, especially during reoxygenation of anoxic tissues.

\section{Reductive pathway of no synthesis}

Nitrate reductase located in the cytosol, which catalyzes reduction of nitrate to nitrite is encoded by two genes in Arabidopsis designated nitrate reductase NADH 1 (NIA1) and NIA2 with NIA2encoding the enzyme which is responsible for NR activity. This enzyme has also potential to catalyze the reduction of nitrite to $\mathrm{NO}^{28,29}$ Various reports provide knowledge in the respect of role for NR in the generation of NO in various cellular processes respectively, stomatal closure, osmotic stress; the plant defence response and auxin reduced lateral root formation. ${ }^{36}$ A plasma membrane-bound NiNOR activity was first described in tobacco, with activity being limited to the roots. The nitrite as substrate for NiNOR is probably provided by plasma membrane-bound NR in a coupled reaction. This enzyme generates extracellular NO and has been suggested to play a role in the sensing nitrate availability and during interactions with mycorrhizal fungi. Unfortunately, the identity of NiNOR still remains to be determined. NO can also be generated by nitrite reduction in the mitochondrial inner membrane, probably via cytochrome c oxidase and/or reductase. ${ }^{37}$ However, this only occurs when the oxygen concentration drops below $20 \mathrm{mM} .{ }^{29} \mathrm{NAD}(\mathrm{P}) \mathrm{H}$ provides electrons via ubiquinone and the mitochondrial electron transport chain. This process has also been reported to produce small amounts of ATP. The peroxisomal enzyme xanthine oxidoreductase (XOR) can also reduce nitrite to NO. XOR has been shown to reduce nitrite to NO, using NADH or xanthine as the reducing substrate. However, this reaction only occurs under anaerobic conditions. As peroxisomes are a major site for the generation of ROIs, this organelle may provide an important location for the interaction of these species with RNIs ${ }^{32}$ (Figure 2). 


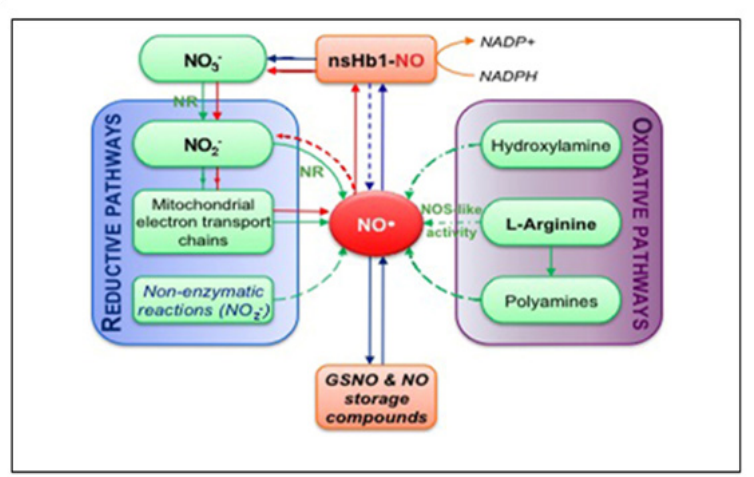

Figure 2 Simplified overview of NO biosynthesis and homeostasis in plant. Source:Arc et al.42

\section{No signals transduction pathway mechanism and function}

Numerous genomic and proteomic methods have been spontaneously used to explicate meticulously NO dependent processes. Principle signal mechanisms convey their bioactivity between covalent modification of NO and RNIs with specific atoms of target proteins. ${ }^{38}$ The post translation modification process of S-nitrolylation, a redox modification of cysteine thiol group by NO exhibit a low pka sulfhydral group which supports significant susceptibility to a range of redox based post translational modification. The modification of these highly reactive Cys residue by NO and related RNIs are reversible except for sulfonic acid formation, the most highly oxidized modification (Figure 3). An experiment has set up to identify protein is regulated by $\mathrm{S}$-nitrosylation in potato tissues. In this experiment, modified and optimize biotin switch assay and nano liquid chromatography combined with mass spectrometry was applied. This modified method provides a new dimension to better understand the signal transduction pathway to derive by NO transient signal. The first in planta biological function for S-nitrosylaltion emerged through a genetics approach, which uncovered a central role for SNOs in plant disease resistance. The exogenous addition of NO donors to plant protein extracts also demonstrated the in vitro formation of plant SNOs. ${ }^{39}$ The list of Snitrosylated plant proteins are currently growing exponentially through the judicious application of the biotin-switch technique. For example, proteins specifically S-nitrosylated during plant immune function, ${ }^{9}$ cold treatment, heavy metal exposure and salt stress have been described. Unfortunately, current strategies for the identification of Cys redox switches on a global scale are not straightforward and typically lack sensitivity. However, new techniques are evolving to help achieve this. ${ }^{40}$ Another pressing current limitation in this area is the sensitivity of the biotin-switch and associated mass spectrometry methodology. In an interesting way, a unique prototype of NOS inhibitor was designed, known by Nanoshutter (NSI), which target the NADPH site of NOS and produces a specific florescence enhancement upon binding to constitutive NOS. The authors proposed that NSI is a promising tool with two photon excitation in the $800-9500 \mathrm{~nm}$ range 9. ${ }^{41} \mathrm{~S}$-nitrosylated proteins are being identified at an increasing rate; deep insights into how these modifications might regulate protein function at the angstrom level are only just beginning to be obtained within a plant biology context. A primer for these studies was the recent demonstration of how S-nitrosylation of an NADPH oxidase,
Respiratory burst oxidase homolog D (RBOHD), modulates the function of this key enzyme. ${ }^{10}$ Therefore, increasingly, NO-oriented research programs may need to embrace structural biology-based approaches.

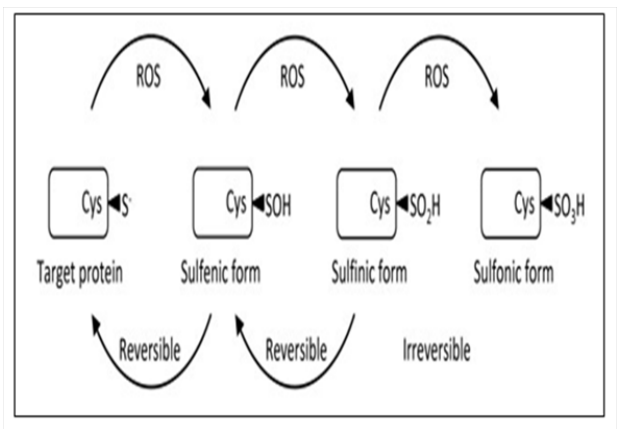

Figure 3 Modification of proteins by ROS.

Source: Li et al.

\section{Conclusion}

Nitrate reductase located in the cytosol, which catalyzes reduction of nitrate to nitrite is encoded by two genes in Arabidopsis designated nitrate reductase NADH 1 (NIA1) and NIA2 with NIA2encoding the enzyme which is responsible for NR activity.

\section{Acknowledgements}

Authors are thankful to Department of Agronomy, School of Agricultures, Lovely Professional University, Jalandhar, for providing the consistent encouragement and undivided attention to the authors.

\section{Conflict of interest}

Author declares there is no conflict of interest.

\section{References}

1. Klepper L. Nitric oxide (NO) and nitrogen dioxide $\left(\mathrm{NO}_{2}\right)$ emissions from herbicide-treated soybean plants. Atmospheric Environment. 1979;13(4):537-542.

2. Noritake T, Kawakita K, Doke N. Nitric oxide induces phytoalexin accumulation in potato tuber tissues. Plant \& Cell Physiology. 1996;37(1):113-116.

3. Stryer, Lubert. Biochemistry, 4th ed. WH. Freeman and Company. 1995. $732 \mathrm{p}$.

4. Wink DA, Mitchell JB. Chemical biology of nitric oxide: Insights into regulatory, cytotoxic, and cytoprotective mechanisms of nitric oxide. Free Radic Biol Med. 1998;25(4-5):434-456.

5. Delledonne M, Zeier J, Marocco A, et al. Signal interactions between nitric oxide and reactive oxygen intermediates in the plant hypersensitive disease resistance response. Proceedings of the National Academy of Sciences. 1998;98(23):13454-13459.

6. Durner J, Wendehenne D, Klessig DF. Defensegeneinductionintobaccoby nitric oxide, cyclic GMP, and cyclic ADP-ribose. Proc Natl Acad of Sci USA. 1998;95(17):10328-10333.

7. Thomas DD, Liu X, Kantrow SP, et al. The biological lifetime of nitric oxide: Implications for the perivascular dynamics of $\mathrm{NO}$ and $\mathrm{O}_{2}$. Proc Natl Acad Sci. 2001;98(1):355-360. 
8. Hong JK, Yun BW, Kang JG, et al. Nitric oxide function and signaling in plant disease resistance. J Exp Bot. 2008;59(2):147-154.

9. Astier J, Lindermayr C. Nitric oxide-dependent posttranslational modification in plants: an update. Int J Mol Sci. 2012;13(11):1519315208.

10. Yun BW, Feechan A, Yin M, et al. S-nitrosylation of NADPH oxidase regulates cell death in plant immunity. Nature. 2011;478(7368):264-268.

11. Camejo D, Romero-Puertas MDC, Rodriguez-Serrano M, et al. Salinityinduced changes in S-nitrosylation of pea mitochondrial proteins. $J$ Proteomics. 2013;79:87-99.

12. Spoel SH, Loake GJ. Redox-based protein modifications: the missing link in plant immune signalling. Current Opinion in Plant Biology. 2011;14(4):358-364.

13. Yu MD, Yun BW, Spoel SH, et al. A sleigh ride through the SNO: regulation of plant immune function by protein $\mathrm{S}-$ nitrosylation. Curr. Opin. Plant Biol. 2012;15(4):424-430.

14. Beligni MV, Lamattina L. Nitric oxide stimulates seed germination and de-etiolation, and inhibits hypocotyl elongation, three light-inducible responses in plants. Planta. 2000;210(2):215-221.

15. Yamasaki H, Sakihama Y, Takahashi S. An alternative pathway for nitric oxide production in plants: new features of an old enzyme. Trends in Plant Science. 1999;4(4):128-129.

16. Clark $\mathrm{G}, \mathrm{Wu} \mathrm{M}$, Wat $\mathrm{N}$, et al. Both the stimulation and inhibition of root hair growth induced by extracellular nucleotides in Arabidopsis are mediated by nitric oxide and reactive oxygen species. Plant Mol Biol. 2000;74(4-5):423-435.

17. Foissner I, Wendehenne $\mathrm{D}$, Langebartels $\mathrm{C}$, et al. In vivo imaging of an elicitor-induced nitric oxide burst in tobacco. Plant J. 2000;23(6):817824.

18. Neill SJ, Desikan R, Clarke A. Hydrogen peroxide and nitric oxide as signalling molecules in plants. J Exp Bot. 2002;53(372):1237-1247.

19. Godber BL, Doel JJ, Sapkota GP, et al. Reduction of nitrite to nitric oxide catalyzed by xanthine oxidoreductase. J Biol Chem . 2000;275(11):77577763.

20. Loake G, Grant M. Salicylic acid in plant defence - the players and protagonists. Curr Opin Plant Biol. 2007;10(5):466-472.

21. Fewson CA, Nicholas DJ. Utilization of nitriv oxide by micro-organisms and higher plants. Nature. 1960;188:794-796.

22. Cooney RV, Harwood PJ, Custer LJ, et al. Light-mediated conversion of nitrogen-dioxide to nitric oxide by carotenoids. Environ Health Perspect. 1994;102(5):460-462.

23. Bethke PC, Gubler F, Jacobsen JV. Dormancy of Arabidopsis seeds and barley grains can be broken by nitric oxide. Planta. 2004;219(5):847-55.

24. Wilkinson JQ, Crawford NM. Identification of the Arabidopsis CHL3 gene as the nitrate reductase structural gene NIA2. Plant Cell. 1991;3(5):461471.

25. Lombardo MC, Lamattina L. Nitric oxide is essential for vesicle formation and trafficking in Arabidopsis root hair growth. J Exp Bot. 2012;63(13):4875-4885.

26. Lopez LC, Escames G, Tapias V, et al. Identification of an inducible nitric oxide synthase in diaphragm mitochondria from septic mice: its relation with mitochondrial dysfunction and prevention by melatonin. Int J Biochem Cell Biol. 2006;38(2):267-278.
27. Escames G, Lopez LC, Ortiz F, et al. Attenuation of cardiac mitochondrial dysfunction by melatonin in septic mice. FEBS J. 2007;274(8):21352147.

28. Yamasaki H, Sakihama Y. Simultaneous production of nitric oxide and peroxynitrite by plant nitrate reductase: In vitro evidence for the NR-dependent formation of active nitrogen species. FEBS Lett. 2000;468(1):89-92.

29. Planchet E, Jagadis Gupta K, Sonoda M, et al. Nitric oxide emission from tobacco leaves and cell suspensions: rate limiting factor sand evidence for the involvement of mitochondrial electron transport. Plant J. 2005;41(5):732-743.

30. Zhou L, Zhu DY. Neuronal NOS: Structure subcellular localization, regulation and clinical implications. Nitric Oxide. 2009;20(4):223-230.

31. Fröhlich A, Durner J. The hunt for plant nitric oxide synthase (NOS): is one really needed? Plant Sci. 2011;181(4):401-404.

32. Del Rio LA, Corpas FJ, Barroso JB. Nitric oxide and nitric oxide synthase activity in plants. Phytochemistry. 2004;65(7):783-792.

33. Tan J, Zhuo C, Guo Z. Nitric oxide mediates cold- and dehydrationinduced expression of a novel MfHyPRP that confers tolerance to abiotic stress. Physiol. Plant. 2013;149(3):310-320.

34. Groppa MD, Rosales EP, Iannone MF, et al. Nitric oxide, polyamines and Cd-induced phytotoxicity in wheat roots. Phytochemistry. 2008;69(14):2609-2615.

35. Arasimowicz-Jelonek M, Floryszak-Wieczorek J, Kubis J. Interaction between polyamine andnitric oxide signaling inadaptive responses to drought in cucumber. Journal of Plant Growth Regulation. 2009;28(2):177-186.

36. Yamamoto-Katou A, Katou S, Yoshioka H, et al. Nitrate reductase is responsible for elicitin-induced nitric oxide production in Nicotiana benthamiana. Plant Cell Physiol. 2006;47(6):726-735.

37. Astier J, Besson-Bard A, Lamotte O, et al. Nitric oxide inhibits the ATPase activity of the chaperone-like AAA+ ATPase CDC48, a target for S-nitrosylation in cryptogein signalling in tobacco cells. Biochem J. 2012a;447(2):249-260.

38. Nathan C. Specificity of a third kind: reactive oxygen and nitrogen intermediates in cell signaling. J Clin Invest. 2003;111(6):769-778.

39. Lindermayr C, Saalbach G, Durner J. Proteomic identification of Snitrosylated proteins in Arabidopsis. Plant Physiol. 2005;137(3):921-930.

40. Fomenko DE, Xing W, Adair BM, et al. High-through put identification of catalytic redox-active cysteine residues. Science. 2007;315(5810):387389.

41. Alderton WK, Cooper CE, Knowles RG. Nitric oxide synthases: structure, function and inhibition. Biochem J. 2001;357(Pt 3):593-615.

42. Arc E, Galland M, Cueff G, et al. Reboot the system thanks to protein posttranslational modifications and proteome diversity: how quiescent seeds restart their metabolism to prepare seedling establishment. Proteomics. 2011;11(9):1606-1618. 